\title{
Reactions of amidrazones with 1,4-quinones
}

\author{
Ashraf A. Aly, ${ }^{*}$ Mohsen A.-M. Gomaa, Ahmed M. Nour El-Din, and Magda S. Fahmy \\ Chemistry Department, Faculty of Science, El-Minia University, 61519-El-Minia, Egypt \\ E-mail: ashraf160@yahoo.com
}

\begin{abstract}
Syntheses of various benzo- and naphtho-1,2,4-triazin-6(4H)-ones are formed in one step via the reactions of amidrazones with benzo- and naphtho-1,4-quinones. In contrast, the reactions of amidrazones with 2,3,5,6-tetrachloro-1,4-benzoquinone or 2,3-dichloro-1,4-naphthoquinone produced the same indazoles, no matter what substituents were present on the nitrogen of the amidrazone.
\end{abstract}

Keywords: Amidrazones, 1,4-quinones, fused 1,2,4-triazin-6(4H)-ones, [3+2]cycloaddition, indazoles

\section{Introduction}

Benzo- and naphth-annulated 1,2,4-triazines are important as pharmaceuticals and agrochemicals. An example is 3-amino-1,2,4-benzotriazine 1,4-dioxide, which has received considerable attention as a new class of antitumor agent. ${ }^{1,2}$ The mechanism of DNA cleavage by this type of compound is therefore of biochemical and pharmaceutical interest. ${ }^{3-5}$ Known syntheses of this azaaromatic framework involve the reaction of 2-nitroaniline with cyanamide, ${ }^{6}$ the base-induced cyclization of 2-nitrophenylurea followed by successive treatment with phosphoryl chloride and gaseous ammonia, ${ }^{7}$ and the addition of diisocyanamide to benzofuroxan followed by acidic work-up. ${ }^{8}$ Some time ago, a convenient synthesis of 3-amino-1,2,4benzotriazine derivatives by the reaction of $o$-fluoronitro-benzenes with free guanidine base was developed. ${ }^{9}$ Amidrazones are an important class of amidines. ${ }^{10}$ Amidrazones display fungistatic, bacteriostatic, and antimycotic activity ${ }^{11}$ and also function as herbicides ${ }^{12}$ and lipoxygenase-1 inhibitors. ${ }^{13}$ In addition, amidrazones can also used to prepare 1,2,4-triazines. ${ }^{14,15}$ 2-Pyridylcarboxamidrazones showed antimicrobacterial activity ${ }^{16}$ and anticancer activity. ${ }^{17}$ Interestingly, $\alpha, \beta$-unsaturated $N, N$-dimethylhydrazones can react with quinones at the electron rich $\delta$-position to form indoles and benzofurans. ${ }^{18,19} \alpha, \beta$-Diketoesters react with amidrazones to yield triazines, generally in good yields. ${ }^{20}$ Treatment of amidrazones with alkyl ketones under acidic catalysis lead generally to 4,5-dihydro- $1 H$-1,2,4-triazoles. ${ }^{21}$ Nitrosation of $N$-formyl amidrazones with 
sodium nitrite in aqueous hydrochloric acid gives tetrazoles in good yields. ${ }^{22}$ We have investigated the reaction of 2,3-diphenylcyclopropenone with $N$-imidoyl-thioureas as amidine analogues. The reaction was interpreted as a stepwise addition to produce pyrimidin- $4(3 H)$ ones. $^{23}$ Aly et al. have recently reported the syntheses of various naphtha-1,2,4-triazepinediones from the reaction of amidrazones with 1,4-dioxo-1,4-dihydronaphthalene-2,3-dicarbonitrile. ${ }^{24}$ Amidrazones also react with 2-(1,3-dioxo-indan-2-ylidene)malononitrile to produce 1,2,4triazoles. $^{25}$ In this paper, we describe a straightforward synthesis of benz- and naphth-annulated 1,2,4-triazines in a single step via the reaction of amidrazones with 1,4-quinones. Also described are some reactions of amidrazones with electron-deficient quinones, which follow a different reaction path.

\section{Results and Discussion}

Amidrazones 1a-d reacted with two equivalents of 1,4-benzoquinone (2) or 1,4-naphthoquinone (5) in absolute ethyl acetate under a $\mathrm{N}_{2}$ atmosphere to yield compounds 3a-d (66-85\%) and 6a-d (70-86\%), respectively, after chromatographic purification and recrystallization (Scheme 1). We chose amidrazones 1a-d having aryl groups with either electron-donating or -withdrawing substitutents on the benzene ring, in order to examine their effect on the reaction. Elemental analyses and IR, NMR $\left({ }^{1} \mathrm{H}\right.$ and $\left.{ }^{13} \mathrm{C}\right)$ and mass spectra were in good agreement with the assigned product structures. For example, the IR spectrum of the benzotriazin-6-one 3a had characteristic triazine- $\mathrm{C}=\mathrm{N}$ bands at $\mathrm{v}=1610$ and 1600, and a carbonyl at $v=1690 \mathrm{~cm}^{-1}$, but no $\mathrm{NH}$ absorption. The elemental analysis and mass spectrum of 3a proved its molecular formula as $\mathrm{C}_{19} \mathrm{H}_{13} \mathrm{~N}_{3} \mathrm{O}$. The ${ }^{1} \mathrm{H}$ NMR spectrum of compound 3a showed the presence of benzotriazine H-5, 7 and -8 at $\delta=6.60(\mathrm{~d}, J=1.0 \mathrm{~Hz}), 6.80(\mathrm{dd}, J=8.0,1.2 \mathrm{~Hz})$ and $7.12(\mathrm{~d}, J=8.0 \mathrm{~Hz})$, respectively. The ${ }^{1} \mathrm{H}$ NMR spectrum of $3 \mathbf{b}$ contained a methoxy singlet at $\delta=3.95$ and benzotriazine signals at $\delta=6.64(\mathrm{~d}, J=1.2 \mathrm{~Hz}$; H-5), $6.86(\mathrm{dd}, J=8.0,1.2 \mathrm{~Hz} ; \mathrm{H}-7)$, and 7.14 (d, $J=8.0 \mathrm{~Hz} ; \mathrm{H}-8)$. The ${ }^{13} \mathrm{C}$ NMR spectra of 3a-d supported the ${ }^{1} \mathrm{H}$ NMR spectral data. For example in 3a, the carbon signals of C-3 and C-8a appeared at $\delta=158.0$ and 156.4, respectively.

Direct one-bond attached hydrogen-carbon correlations were established by ${ }^{1} \mathrm{H},{ }^{13} \mathrm{C}$ - COSY (HETCOR), which correlated the aromatic carbons with their protons in both 3a and 6a. In 3a, there is a correlation between C-6 and quinonoid protons $\mathrm{H}-5,-7$ and -8 . In the case of 6a, the mass spectrum and elemental analysis established its molecular formula as $\mathrm{C}_{23} \mathrm{H}_{15} \mathrm{~N}_{3} \mathrm{O}$. The $\mathrm{H}-5$ proton in 6a resonated at $\delta=6.80$, and $\mathrm{C}-5$ appeared in the ${ }^{13} \mathrm{C}$ NMR spectrum at $\delta=110.0$. The ${ }^{13} \mathrm{C}$ NMR spectrum also showed C-3, $-6,-6 \mathrm{a}$, and $-10 \mathrm{a}$ at $\delta=158.8,185.0,132.0$, and 134.0, respectively. Selected chemical shifts of compounds 3a and 6a are shown in Figure 1. 


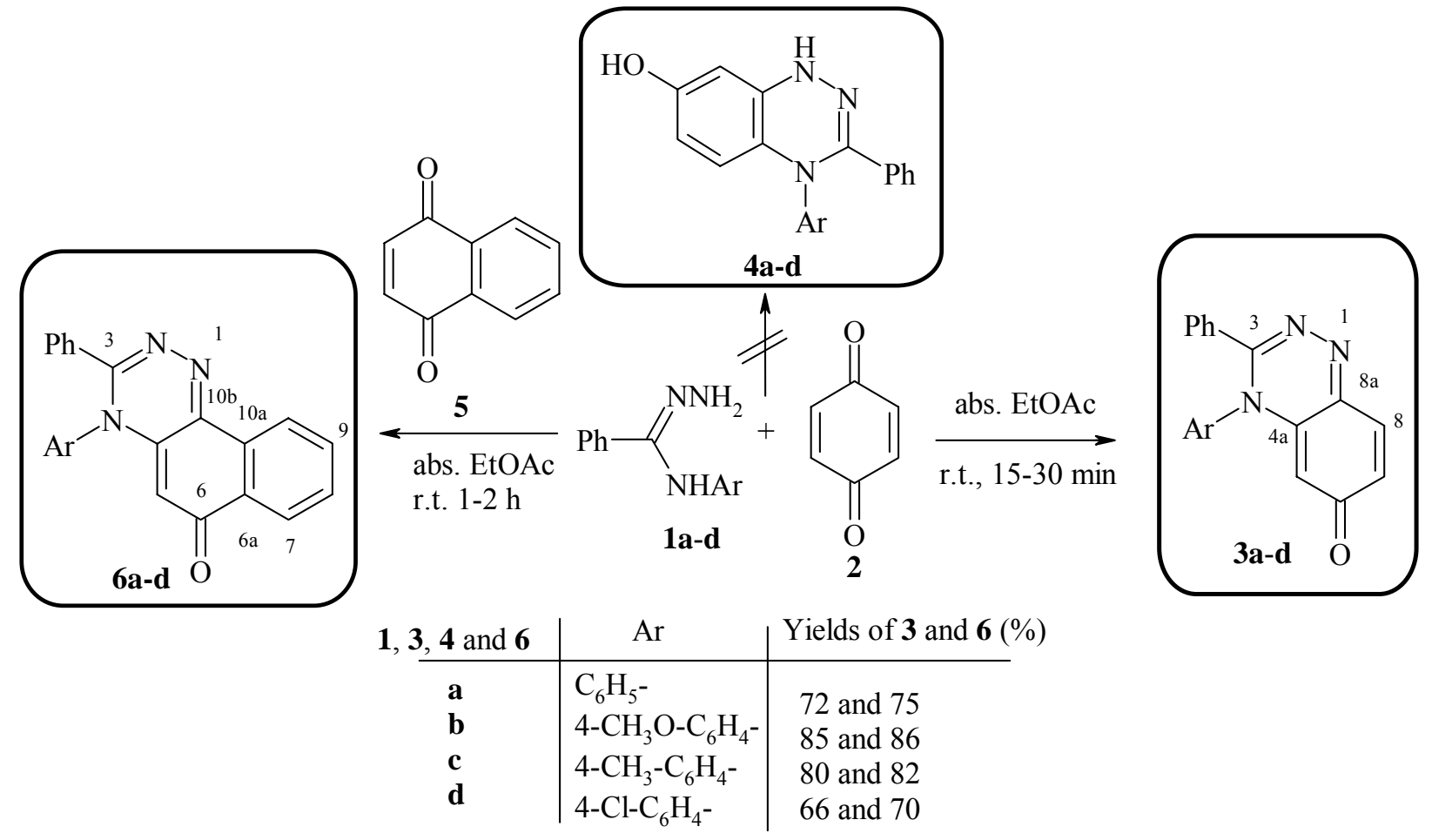

Scheme 1. Synthesis of benzo- and naphtha-1,2,4-triazin-6(4H)-ones 3a-d and 6a-d.

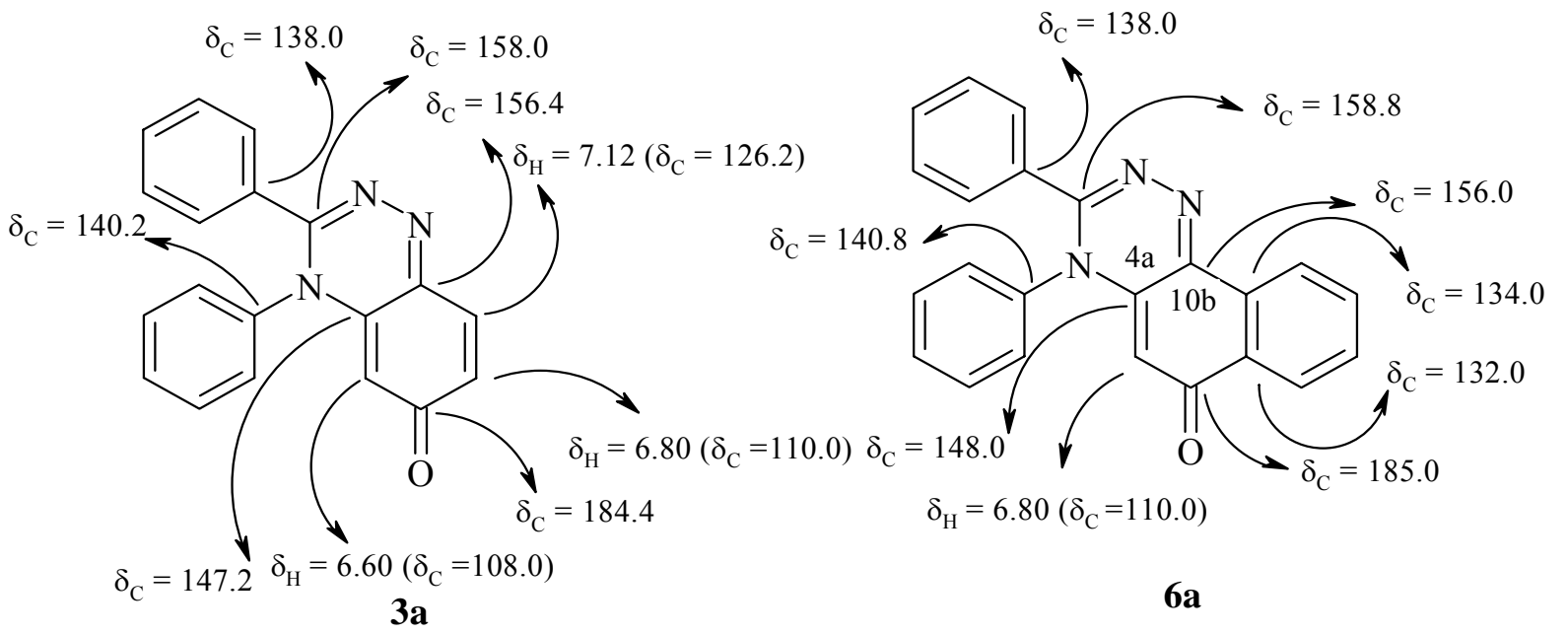

Figure 1. Distinctive $\delta$ ' values of compounds 3a and 6a.

We believe that the hydrazino- $\mathrm{NH}_{2}$ group is more nucleophilic than the aromatic amine and that the formation of 3a-d (or 6a-d) can be rationalized by initial nucleophilic attack of the terminal hydrazino nitrogen of 1a-d at one carbonyl group of $\mathbf{2}$ to form intermediate 7, followed by elimination of water to produce $\mathbf{8}$ (Scheme 2). A further nucleophilic amidine-like addition at $\mathrm{C}-2$ of the quinone would form the salt $\mathbf{9}$. Finally, we suggest that intermediate 9 is oxidized by 
another mol of 2 to give 3a-d (Scheme 2). Isolation of 1,4-dihydro-benzo- and/or naphthoquinones supports the proposed mechanism. Spectroscopic data proved unambiguously the structure of compounds 3a-d and excluded any other posssibilities such the formation of compounds 4a-d (Scheme 1). Since amidrazones can be described as amino derivatives of $N$ substituted hydrazones, ${ }^{26}$ the proposed mechanism is supported by literature precedent, which indicated that aromatic amines can react with 1,2- and 1,4-benzoquinones via condensation and elimination processes. ${ }^{27}$

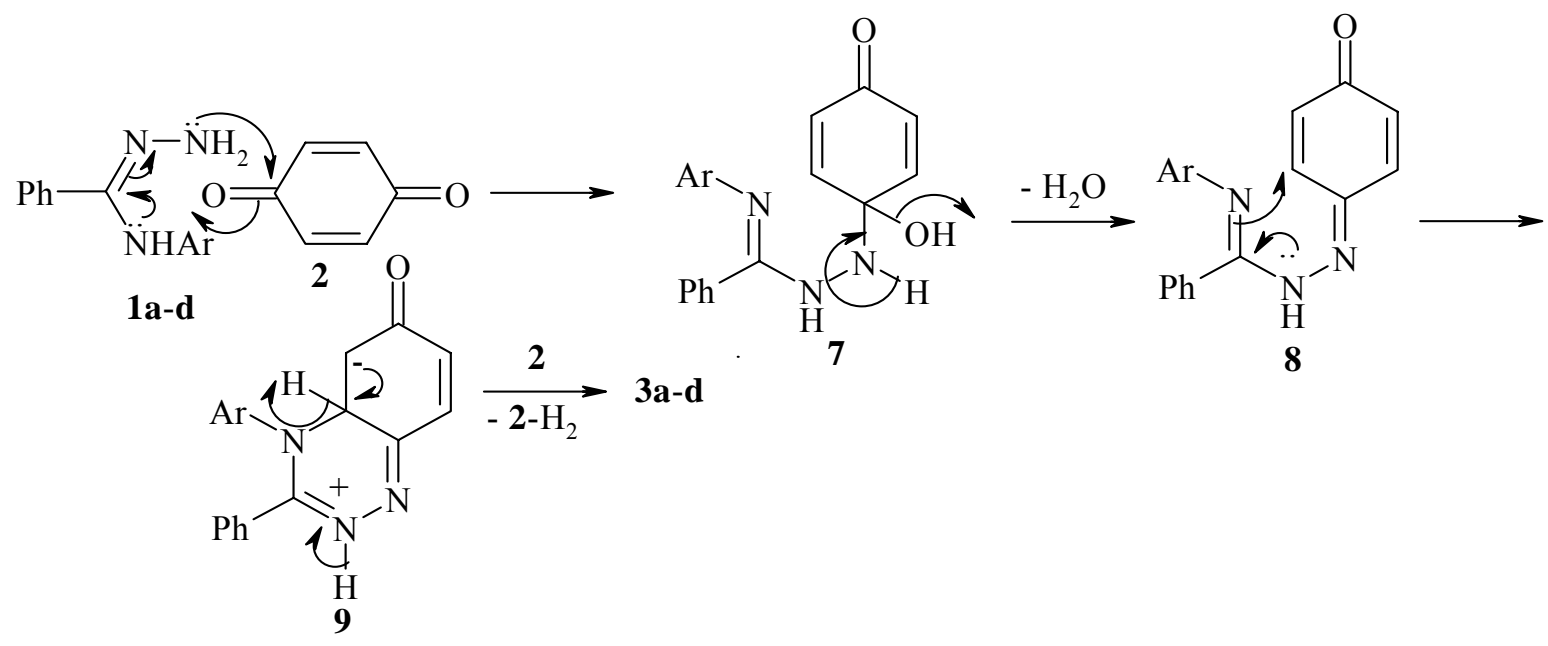

Scheme 2. Rational formation of triazin-6(4H)-ones 3a-d.

In the light of the aforementioned results, our attention turned to the reactions of 1a-d with 2,3,5,6-tetrachloro-1,4-benzoquinone (10) and 2,3-dichloro-1,4-naphthoquinone (15) (Scheme 3) in dry DMF. The reaction of each of $\mathbf{1 a - d}$ with $\mathbf{1 0}$ produced the same product, the spectroscopic and analytical data for which showed it to be 5,6-dichloro-3-phenyl- $1 \mathrm{H}$-indazole-4,7-dione (12) (Scheme 3). In the same manner, the reaction of 1a-d with 15 all yielded 16, which was identified as 3-phenyl-1H-benzo[f]indazole-4,9-dione (16) (Scheme 3). Mass spectrometric and elemental analysis of $\mathbf{1 2}$ proved the molecular formula to be $\mathrm{C}_{13} \mathrm{H}_{6} \mathrm{Cl}_{2} \mathrm{~N}_{2} \mathrm{O}_{2}$. The IR spectrum of 12 had two broad peaks at $v=3220$ and $1700-1682 \mathrm{~cm}^{-1}$ assigned to the $\mathrm{NH}$ and carbonyl groups, respectively. The ${ }^{1} \mathrm{H}$ NMR spectrum of $\mathbf{1 2}$ showed a broad singlet at $\delta=12.40$. From analytical and spectroscopic data, it could be concluded that the reaction between 1a-d and $\mathbf{1 0}$ was accompanied by the overall loss of one molecule of $\mathrm{Cl}_{2}$ and one of the corresponding aromatic amine. In the ${ }^{13} \mathrm{C}$ NMR spectrum of 12, the two carbonyl carbons resonated at $\delta=175.8$ and 176.4; C-5 and -6 resonated at $\delta=124.5$ and 126.8; and C-3 and -3 a appeared at $\delta=155.8$ and 132.0. One might have also expected the formation of triazines 13a-d or 14a-d (Scheme 1), however, this possibility is excluded by the spectroscopic data discussed above. 


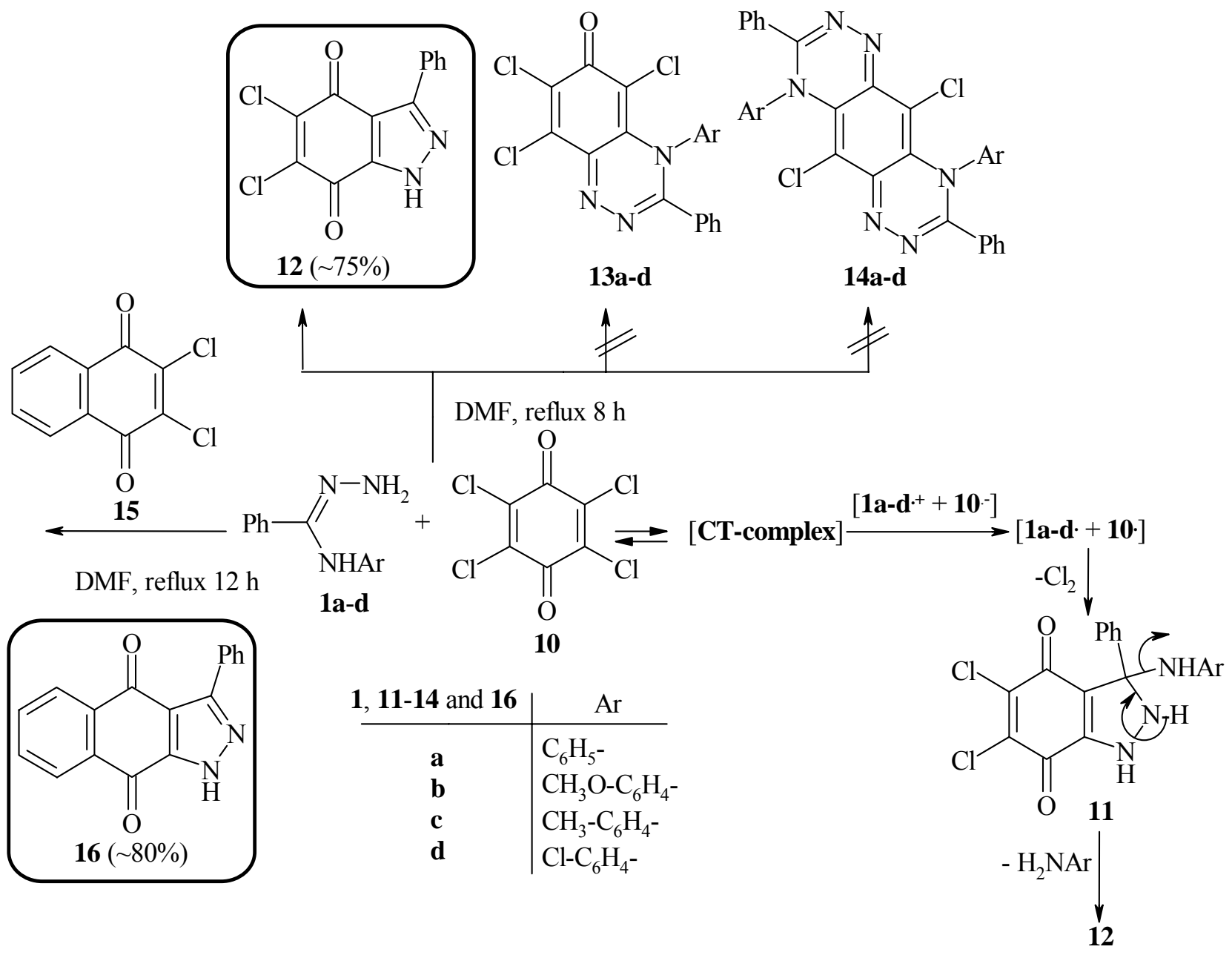

Scheme 3. Synthesis of indazoles 12 and 16.

The structure assignment of $\mathbf{1 6}$ is based on analytical and spectral data: its IR spectrum contained an $\mathrm{NH}$ signal at about $v=3200 \mathrm{~cm}^{-1}$, in addition to broad carbonyl absorption at $v=$ $1700-1685 \mathrm{~cm}^{-1}$. The NMR spectra of $\mathbf{1 6}$ are in accordance to the proposed structure (Scheme 3) thus the ${ }^{13} \mathrm{C}$ NMR spectrum contained signals for C-3, -4 and -9 at $\delta=156.0,175.8$ and 176.8, respectively (see also the Experimental Section). We suggest that formation of these products can be rationalized by the mechanism shown in Scheme 3: an unstable CTC is formed, in the case of reaction between 1a-d with 10, followed by the formation of radicals $\mathbf{1 a - \mathbf { d } ^ { + }}$ and $\mathbf{1 0}^{-}$ (Scheme 3). Combination of the two radicals and extrusion of a chlorine molecule would form 11 (Scheme 3). Finally, elimination of a molecule of arylamine from $\mathbf{1 1}$ would give the observed product 12 (Scheme 3). Similar reactivity between donors and acceptors (i.e. 11 and 15) has been recently discussed by us. ${ }^{28}$

In conclusion, our method is a convenient procedure to synthesize fused 1,2,4-triazines. Its advantages are the reasonable yields, and the ease with which the reaction can be carried out as a 
one-pot procedure. Additionally, amidrazones react with halogenated quinones to give indazoles. possibly via CT-complexes.

\section{Experimental Section}

General Procedures. Melting points are uncorrected. ${ }^{1} \mathrm{H} \mathrm{NMR}$ and ${ }^{13} \mathrm{C}$ NMR spectra (Bruker $\mathrm{AM} 400,{ }^{1} \mathrm{H}: 400.13 \mathrm{MHz},{ }^{13} \mathrm{C}: 100.6 \mathrm{MHz}$ ) were obtained from $\mathrm{CDCl}_{3}$ solutions; the chemical shifts are given relative to internal standard TMS. For preparative thin layer chromatography (PLC), glass plates $(20 \times 48 \mathrm{~cm})$ were covered with a slurry of silica gel Merck $\mathrm{PF}_{254}$ and air dried and developed using the solvents listed. Zones were detected by quenching of indicator fluorescence upon exposure to $254 \mathrm{~nm}$ UV light. Elemental analyses were carried in the Assiut Microanalysis Center of Assiut University. Mass spectrometry was performed by electron impact at $70 \mathrm{eV}$, with a Finnigan Mat 8430 spectrometer in the Institute of Organic Chemistry, TUBraunschweig. Germany. IR spectra were run on a Shimadzu 470 spectrometer using $\mathrm{KBr}$ pellets. Ultraviolet (UV) spectra were obtained of acetonitrile solutions, on a Perkin Spectrometer.

Starting materials. Amidrazones 1a-d were prepared according to reference 10. Benzo- and naphtho-1,4-quinones (2 and 5) were obtained from Fluka, whereas 2,3,5,6-tetrachloro-1,4benzoquinone (10) and 2,3-dichloro-1,4-naphthoquinone (15) were obtained from Aldrich.

\section{Reaction of amidrazones 1a-d with 2 and 5}

General procedure. A $250 \mathrm{ml}$ two-necked round-bottom flask was flame-dried under $\mathrm{N}_{2}$ and then cooled to room temperature. Under an $\mathrm{N}_{2}$ atmosphere, dry absolute ethyl acetate $(30 \mathrm{~mL})$ containing a mixture of 1a-d (1 mmol) and 2 (or 5 ) $(2 \mathrm{mmol})$ was added. The mixture was stirred at room temperature (the reaction was followed by TLC analysis). The solvent was then removed under vacuum and the residue was separated by preparative plates chromatography (silica gel, toluene: ethyl acetate 5:2). The obtained products 3a-d or 6a-d were isolated as the fastest migrating zones, whereas 1,4-dihydro benzo- and/or naphtho-quinone as the slowest zones. The products were recrystallized from the stated solvents, whereas the dihydroquinones were identified by comparison using authentic samples by TLC analyses along with m.p. measurements.

3,4-Diphenyl-1,2,4-benzotriazin-6(4H)-one (3a). Orange crystals $(0.22$ g. $72 \%) ; R_{f}=0.4$ $\left(\mathrm{CH}_{2} \mathrm{Cl}_{2}\right)$, m.p. $238^{\circ} \mathrm{C}$ (ethyl acetate). - IR (KBr): $v=3060-3010(\mathrm{Ar}-\mathrm{CH}), 1690(\mathrm{C}=\mathrm{O}), 1610$, $1600(\mathrm{C}=\mathrm{N}), 1590(\mathrm{C}=\mathrm{C}) \mathrm{cm}^{-1}$. - UV $\left(\mathrm{CH}_{3} \mathrm{CN}\right): \lambda_{\max }(\log \varepsilon)=410(4.10) .-{ }^{1} \mathrm{H} \mathrm{NMR}\left(\mathrm{CDCl}_{3}\right): \delta$ $=6.60(\mathrm{~d}, 1 \mathrm{H}, J=1.0 \mathrm{~Hz}, \mathrm{H}-5), 6.80(\mathrm{dd}, 1 \mathrm{H}, J=8.0,1.2 \mathrm{~Hz}, \mathrm{H}-7), 7.12(\mathrm{~d}, 1 \mathrm{H}, J=8.0 \mathrm{~Hz}, \mathrm{H}-$ 8), 7.20-7.28 (m, $3 \mathrm{H}, \mathrm{Ar}-\mathrm{H}), 7.43-7.60$ (m, $5 \mathrm{H}, \mathrm{Ar}-\mathrm{H}), 7.66-7.70$ (m, $2 \mathrm{H}, \mathrm{Ar}-\mathrm{H}) .-{ }^{13} \mathrm{C}$ NMR $\left(\mathrm{CDCl}_{3}\right): \delta=108.0(\mathrm{CH}-5), 110.0(\mathrm{CH}-7), 126.2(\mathrm{CH}-8), 126.8,127.2(p-\mathrm{Ar} \mathrm{CH}), 127.8,128.0$, 128.4, 128.6 (Ar 2 CH), 138.0 (Ph C), 140.2 (N-Ph C), 147.2 (C-4a), 156.4 (C-8a), 158.0 (C-3), 
184.4 (C-6). - MS (EI, $70 \mathrm{eV}): \mathrm{m} / \mathrm{z}(\%)=299\left[\mathrm{M}^{+}\right]$(100), 295 (20), 257 (18), 222 (42), 194 (20), 180 (60), 178 (30), 118 (24), 91 (26), 77 (44), 56 (14). - $\mathrm{C}_{19} \mathrm{H}_{13} \mathrm{~N}_{3} \mathrm{O}$ (299.33): Calcd. C, 76.24; H, 4.38; N, 14.04. Found: C, 76.14; H, 4.30; N, 14.00.

4-(4-Methoxyphenyl)-3-phenyl-1,2,4-benzotriazin-6(4H)-one (3b). Orange crystals (0.28 g. $85 \%) ; R_{f}=0.5\left(\mathrm{CH}_{2} \mathrm{Cl}_{2}\right)$, m.p. $258^{\circ} \mathrm{C}$ (ethanol). - IR (KBr): $v=3080-3030(\mathrm{Ar}-\mathrm{CH}), 2980-2860$ (aliph-CH), $1692(\mathrm{C}=\mathrm{O}), 1612,1604(\mathrm{C}=\mathrm{N}), 1594(\mathrm{C}=\mathrm{C}) \mathrm{cm}^{-1}$. - UV $\left(\mathrm{CH}_{3} \mathrm{CN}\right): \lambda_{\max }(\log \varepsilon)=$ 420 (4.12). - ${ }^{1} \mathrm{H}$ NMR $\left(\mathrm{CDCl}_{3}\right): \delta=3.95\left(\mathrm{~s}, 3 \mathrm{H}, \mathrm{OCH}_{3}\right), 6.64(\mathrm{~d}, 1 \mathrm{H}, \mathrm{J}=1.2 \mathrm{~Hz}, \mathrm{H}-5), 6.70$ (dd, $\left.2 \mathrm{H}, J=8.0,1.2 \mathrm{~Hz}, \mathrm{OCH}_{3}-\mathrm{Ph}\right), 6.86$ (dd, $\left.1 \mathrm{H}, J=8.0,1.0 \mathrm{~Hz}, \mathrm{H}-7\right), 7.14$ (d, $1 \mathrm{H}, J=8.0 \mathrm{~Hz}, \mathrm{H}-$ 8), 7.40-7.60 (m, $5 \mathrm{H}, \mathrm{Ar}-\mathrm{H}), 7.80-7.90\left(\mathrm{dd}, 2 \mathrm{H}, J=8.0,1.0 \mathrm{~Hz}, \mathrm{OCH}_{3}-\mathrm{Ph}\right) .-{ }^{13} \mathrm{C} \mathrm{NMR}$ $\left(\mathrm{CDCl}_{3}\right): \delta=51.0\left(\mathrm{OCH}_{3}\right), 108.4(\mathrm{CH}-5), 110.6(\mathrm{CH}-7), 126.6(\mathrm{CH}-8), 126.8,(p-\mathrm{Ar} \mathrm{CH}), 127.4$ $\left(\mathrm{OCH}_{3}-\mathrm{Ph} 2 \mathrm{CH}\right), 128.6,128.8(\mathrm{Ar} 2 \mathrm{CH}), 132.0\left(\mathrm{OCH}_{3}-\mathrm{Ph} 2 \mathrm{CH}\right), 137.4\left(\mathrm{OCH}_{3}-\mathrm{Ph} \mathrm{C}\right), 138.6(\mathrm{Ph}$ C), 140.4 (N-Ph C), 147.0 (C-4a), 156.2 (C-8a), 158.4 (C-3), 184.5 (C-6). - MS (EI, 70 eV): m/z $(\%)=330[\mathrm{M}+1](22), 329\left[\mathrm{M}^{+}\right](100), 314$ (20), 298 (40), 252 (28), 238 (24), 221 (26), 212 (32), 185 (36), 144 (36), 118 (32), 109 (26), 78 (40), 56 (24). - $\mathrm{C}_{20} \mathrm{H}_{15} \mathrm{~N}_{3} \mathrm{O}_{2}$ (329.36): Calcd. C, 72.94; H, 4.59; N, 12.76. Found: C, 73.04; H, 4.50; N, 12.70.

4-(4-Methylphenyl)-3-phenyl-1,2,4-benzotriazin-6(4H)-one (3c). Orange crystals (0.25 g. $80 \%) ; R_{f}=0.45\left(\mathrm{CH}_{2} \mathrm{Cl}_{2}\right)$, m.p. $218{ }^{\circ} \mathrm{C}$ (methanol). - IR (KBr): $v=3065-3010(\mathrm{Ar}-\mathrm{CH}), 2986-$ 2870 (aliph-CH), $1690(\mathrm{C}=\mathrm{O}), 1610,1600(\mathrm{C}=\mathrm{N}), 1596(\mathrm{C}=\mathrm{C}) \mathrm{cm}^{-1}$. $-\mathrm{UV}\left(\mathrm{CH}_{3} \mathrm{CN}\right): \lambda_{\max }(\log \varepsilon)$ $=416$ (4.10). $-{ }^{1} \mathrm{H} \mathrm{NMR}\left(\mathrm{CDCl}_{3}\right): \delta=2.38\left(\mathrm{~s}, 3 \mathrm{H}, \mathrm{CH}_{3}\right), 6.60(\mathrm{~d}, 1 \mathrm{H}, J=1.0 \mathrm{~Hz}, \mathrm{H}-5), 6.80(\mathrm{dd}$, $1 \mathrm{H}, J=8.0,1.0 \mathrm{~Hz}, \mathrm{H}-7), 7.10$ (d, $1 \mathrm{H}, J=8.0 \mathrm{~Hz}, \mathrm{H}-8), 7.20-7.40$ (m, $7 \mathrm{H}, \mathrm{Ar}-\mathrm{H}), 7.60-7.68$ $(\mathrm{m}, 2 \mathrm{H}, \mathrm{Ar}-\mathrm{H}) .-{ }^{13} \mathrm{C} \mathrm{NMR}\left(\mathrm{CDCl}_{3}\right): \delta=32.8\left(\mathrm{CH}_{3}\right), 108.4(\mathrm{CH}-5), 110.6(\mathrm{CH}-7), 126.4(\mathrm{CH}-$ 8), 127.0 (p-Ar CH), 128.0, 128.6, 128.8, 130.0 (Ar 2CH), $132.2\left(\mathrm{CH}_{3}-\mathrm{Ph} \mathrm{C}\right), 138.2,140.0(\mathrm{~N}-\mathrm{Ph}$ C), 146.8 (C-4a), 156.0 (C-8a), 158.0 (C-3), 184.2 (C-6). - MS (EI, $70 \mathrm{eV}): \mathrm{m} / \mathrm{z}(\%)=313\left[\mathrm{M}^{+}\right]$ (100), 298 (36), 220 (24), 210 (34), 144 (30), 92 (40), 77 (30), 56 (26). - $\mathrm{C}_{20} \mathrm{H}_{15} \mathrm{~N}_{3} \mathrm{O}$ (313.36): Calcd. C, 76.66; H, 4.82; N, 13.41. Found: C, 76.60; H, 4.78; N, 13.36.

4-(4-Chlorophenyl)-3-phenyl-1,2,4-benzotriazin-6(4H)-one (3d). Pale orange crystals (0.22 g, $66 \%) ; R_{f}=0.25\left(\mathrm{CH}_{2} \mathrm{Cl}_{2}\right)$, m.p. $168{ }^{\circ} \mathrm{C}$ (ethanol). - IR (KBr): $v=3050-3008(\mathrm{Ar}-\mathrm{CH}), 1690$ $(\mathrm{C}=\mathrm{O}), 1606(\mathrm{C}=\mathrm{N}), 1590(\mathrm{C}=\mathrm{C}) \mathrm{cm}^{-1}$. - UV $\left(\mathrm{CH}_{3} \mathrm{CN}\right): \lambda_{\max }(\log \varepsilon)=398(3.8)$. - ${ }^{1} \mathrm{H} \mathrm{NMR}$ $\left(\mathrm{CDCl}_{3}\right): \delta=6.56(\mathrm{~d}, 1 \mathrm{H}, J=1.2 \mathrm{~Hz}, \mathrm{H}-5), 6.70-6.76$ (m, $\left.3 \mathrm{H}, \mathrm{Ar}-\mathrm{H}, \mathrm{H}-7\right), 7.08$ (d, $1 \mathrm{H}, J=8.0$ $\mathrm{Hz}, \mathrm{H}-8), 7.22-7.38$ (m, $7 \mathrm{H}, \mathrm{Ar}-\mathrm{H}) .-{ }^{13} \mathrm{C} \mathrm{NMR}\left(\mathrm{CDCl}_{3}\right): \delta=108.0(\mathrm{CH}-5), 110.0$ (CH-7), 126.2 (CH-8), 126.8 (Cl-Ar 2CH), 127.4 (p-Ar CH), 127.6, 128.0, (Ar 2CH), 128.4 (Cl-Ar C), 128.8 (Cl-Ar 2CH), 138.0 (Ph C), 140.0 (N-Ph C), 146.5 (C-4a), 156.0 (C-8a), 157.4 (C-3), 184.0 (C6). - MS (EI, $70 \mathrm{eV}): \mathrm{m} / \mathrm{z}(\%)=335[\mathrm{M}+2](34), 333\left[\mathrm{M}^{+}\right](100), 300$ (34), 298 (32), 222 (28), 220 (24), 212 (30), 210 (34), 145 (26), 115 (38), 113 (36), 77 (26), 56 (20). - $\mathrm{C}_{19} \mathrm{H}_{12} \mathrm{ClN}_{3} \mathrm{O}$ (333.78): Calcd. C, 68.37; H, 3.62; Cl, 10.62; N, 12.59. Found: C, 68.20; H, 3.68; Cl, 10.55; N, 12.56 .

3,4-Diphenyl-naphtho[2,1-e]-1,2,4-triazin-6(4H)-one (6a). Red crystals $(0.26 \mathrm{~g} 75 \%) ; R_{f}=0.5$ $\left(\mathrm{CH}_{2} \mathrm{Cl}_{2}\right)$, m.p. $288{ }^{\circ} \mathrm{C}$ (ethyl acetate). - IR $(\mathrm{KBr}): v=3040-3000(\mathrm{Ar}-\mathrm{CH}), 1700-1685(\mathrm{C}=\mathrm{O})$, $1600(\mathrm{C}=\mathrm{N}), 1595(\mathrm{C}=\mathrm{C}) \mathrm{cm}^{-1}$. - UV $\left(\mathrm{CH}_{3} \mathrm{CN}\right): \lambda_{\max }(\log \varepsilon)=400(3.8)$. - ${ }^{1} \mathrm{H} \mathrm{NMR}\left(\mathrm{CDCl}_{3}\right): \delta=$ 6.80 (s, 1 H, H-5), 7.18-7.30 (m, 5 H, Ar-H), 7.50-7.60 (m, 7 H, Ar-H), 7.94 (dd, 2 H, J = 8.0, 
$1.2 \mathrm{~Hz}, \mathrm{Ar}-\mathrm{H}) .-{ }^{13} \mathrm{C} \mathrm{NMR}\left(\mathrm{CDCl}_{3}\right): \delta=110.0(\mathrm{CH}-5), 126.6,127.0(p-\mathrm{Ar} \mathrm{CH}), 127.6(\mathrm{Ar}-\mathrm{CH})$, 128.0, 128.2, 128.6, 129.0 (Ar 2CH), 129.4, 130.0, 131.0 (Ar CH), 132.0 (C-6a), 134.0 (C-10a), 134.4 (Ph C), 140.8 (N-Ph C), 148.0 (C-4a), 156.0 (C-10b), 158.8 (C-3), 185.0 (C-6). - MS (EI, $70 \mathrm{eV}): m / z(\%)=349\left[\mathrm{M}^{+}\right](100), 272$ (46), 195 (20), (32), 180 (42), 116 (28), 91 (20), 77 (60), 51 (20). $-\mathrm{C}_{23} \mathrm{H}_{15} \mathrm{~N}_{3} \mathrm{O}$ (349.40): Calcd. C, 79.07; H, 4.33; N, 12.03. Found C, 79.00; H, 4.29; N, 12.00 .

4-(4-Methoxyphenyl)-3-phenyl-naphtho[2,1-e]-1,2,4-triazin-6(4H)-one (6b). Red crystals (0.31 g, 86\%); $R_{f}=0.40\left(\mathrm{CH}_{2} \mathrm{Cl}_{2}\right)$, m.p. $182{ }^{\circ} \mathrm{C}$ (methanol). - IR (KBr): $v=3066-3008(\mathrm{Ar}-\mathrm{CH})$, 2980-2860 (aliph-CH), 1702-1690 (C=O), $1610(\mathrm{C}=\mathrm{N}), 1596(\mathrm{C}=\mathrm{C}) \mathrm{cm}^{-1}$. - UV $\left(\mathrm{CH}_{3} \mathrm{CN}\right): \lambda_{\max }$ $(\log \varepsilon)=436(4.2) .-{ }^{1} \mathrm{H}$ NMR $\left(\mathrm{CDCl}_{3}\right): \delta=3.90\left(\mathrm{~s}, 3 \mathrm{H}, \mathrm{OCH}_{3}\right), 6.82(\mathrm{~s}, 1 \mathrm{H}, \mathrm{H}-5), 6.90(\mathrm{dd}, 2$ $\left.\mathrm{H}, J=8.0,1.0 \mathrm{~Hz}, \mathrm{CH}_{3} \mathrm{OPh}-\mathrm{H}\right), 7.24-7.50(\mathrm{~m}, 7 \mathrm{H}, \mathrm{Ar}-\mathrm{H}), 7.80(\mathrm{dd}, 2 \mathrm{H}, J=8.0,1.2 \mathrm{~Hz}$, $\left.\mathrm{CH}_{3} \mathrm{OPh}-\mathrm{H}\right), 7.98$ (dd, $\left.2 \mathrm{H}, J=8.0,1.2 \mathrm{~Hz}, \mathrm{Ar}-\mathrm{H}\right) .-{ }^{13} \mathrm{C} \mathrm{NMR}\left(\mathrm{CDCl}_{3}\right): \delta=51.9\left(\mathrm{OCH}_{3}\right), 110.4$ (CH-5), 126.4 (p-Ar CH), $127.0(\mathrm{Ar} \mathrm{CH}), 127.2\left(\mathrm{OCH}_{3}-\mathrm{Ph} 2 \mathrm{CH}\right), 128.5,128.8(\mathrm{Ar} 2 \mathrm{CH}), 129.0$, 130.4, 131.2 (Ar CH), $131.6\left(\mathrm{OCH}_{3}-\mathrm{Ph} 2 \mathrm{CH}\right), 132.2$ (C-6a), 134.4 (C-10a), 134.6 (Ph C), 140.4 (N-Ph C), 148.4 (C-4a), $150.0\left(\mathrm{CH}_{3} \mathrm{O}-\mathrm{Ph} \mathrm{C}\right) 156.0$ (C-3), 156.6 (C-10b), 185.5 (C-6). - MS (EI, $70 \mathrm{eV}): m / z(\%)=379\left[\mathrm{M}^{+}\right]$(100), 348 (22), 332 (20), 286 (24), 272 (40), 194 (26), 180 (40), 166 (24), 108 (34), 92 (30), 77 (50), 50 (18). - $\mathrm{C}_{24} \mathrm{H}_{17} \mathrm{~N}_{3} \mathrm{O}_{2}$ (379.42): Calcd. C, 75.98; H, 4.52; N, 11.07. Found C, 75.80; H, 4.50; N, 11.00.

4-(4-Methylphenyl)-3-phenyl-naphtho[2,1-e]-1,2,4-triazin-6(4H)-one (6c). Pale red crystals (0.30 g, 82\%); $R_{f}=0.25\left(\mathrm{CH}_{2} \mathrm{Cl}_{2}\right)$, m.p. $240{ }^{\circ} \mathrm{C}$ (acetonitrile). - IR (KBr): $v=3100-2996$ (Ar$\mathrm{CH}), 2980-2860$ (Aliph-CH), 1700-1684 $(\mathrm{C}=\mathrm{O}), 1600(\mathrm{C}=\mathrm{N}) \mathrm{cm}^{-1}$. - UV $\left(\mathrm{CH}_{3} \mathrm{CN}\right): \lambda_{\max }(\log \varepsilon)=$ 426 (4.1). - ${ }^{1} \mathrm{H}$ NMR $\left(\mathrm{CDCl}_{3}\right): \delta=2.38\left(\mathrm{~s}, 3 \mathrm{H}, \mathrm{CH}_{3}\right), 6.84$ (s, $\left.1 \mathrm{H}, \mathrm{H}-5\right), 7.24-7.36$ (m, $7 \mathrm{H}, \mathrm{Ar}-$ $\mathrm{H})$, 7.50-7.60 (m, $4 \mathrm{H}, \mathrm{Ar}-\mathrm{H}), 7.90(\mathrm{dd}, 2 \mathrm{H}, J=8.0,1.0 \mathrm{~Hz}, \mathrm{Ar}-\mathrm{H}) .{ }^{13} \mathrm{C} \mathrm{NMR}\left(\mathrm{CDCl}_{3}\right): \delta=32.0$ $\left(\mathrm{CH}_{3}\right), 110.6$ (CH-5), 126.6 (p-Ar $\left.\mathrm{CH}\right), 127.2,128.0,128.5,128.8$ (Ar 2CH), 130.0, 130.6, 132.0, 132.4 (Ar CH), 132.8 (C-6a), 133.6 (Ph C), 134.0 (C-10a), $136.0\left(\mathrm{CH}_{3} \mathrm{Ph} \mathrm{C}\right), 140.0(N-\mathrm{Ph}$ C), 148.4 (C-4a), 156.4 (C-3), 158.0 (C-10b), 185.2 (C-6). - MS (EI, 70 eV): m/z (\%) = $296\left[\mathrm{M}^{+}\right]$ (4), 331 (4), 252 (9), 248 (100), 246 (80), 210 (22), 175 (24), 147 (32), 87 (44). - MS (EI, 70 $\mathrm{eV}): \mathrm{m} / \mathrm{z}(\%)=363\left[\mathrm{M}^{+}\right]$(100), 348 (22), 272 (40), 194 (26), 180 (40), 166 (24), 118 (34), 92 (30), 77 (50), 50 (18). - $\mathrm{C}_{24} \mathrm{H}_{17} \mathrm{~N}_{3} \mathrm{O}$ (363.42): Calcd. C, 79.32; H, 4.72; N, 11.56. Found C, 79.20; H, 4.69; N, 11.48 .

4-(4-Chlorophenyl)-3-phenyl-naphtho[2,1-e]-1,2,4-triazin-6(4H)-one (6d). Red crystals (0.25 g, 70\%); $R_{f}=0.5\left(\mathrm{CH}_{2} \mathrm{Cl}_{2}\right)$, m.p. $162{ }^{\circ} \mathrm{C}$ (ethanol). - IR (KBr): $v=3220(\mathrm{NH}), 3070-3000(\mathrm{Ar}-$ $\mathrm{CH}), 1700-1690(\mathrm{CO}), 1600(\mathrm{C}=\mathrm{N}), 1598(\mathrm{C}=\mathrm{C}) \mathrm{cm}^{-1}$. - UV $\left(\mathrm{CH}_{3} \mathrm{CN}\right): \lambda_{\max }(\log \varepsilon)=402(4.0)$. ${ }^{1} \mathrm{H}$ NMR $\left(\mathrm{CDCl}_{3}\right): \delta=6.70(\mathrm{~s}, 1 \mathrm{H}, \mathrm{H}-5), 6.80(\mathrm{dd}, 2 \mathrm{H}, J=8.0,1.0 \mathrm{~Hz}, \mathrm{ClPh}-\mathrm{H}), 7.20-7.50(\mathrm{~m}$, $7 \mathrm{H}, \mathrm{Ar}-\mathrm{H}), 7.60(\mathrm{dd}, 2 \mathrm{H}, J=8.0,1.2 \mathrm{~Hz}), 7.92(\mathrm{dd}, 2 \mathrm{H}, J=8.0,1.0 \mathrm{~Hz}, \mathrm{Ar}-\mathrm{H}) .-{ }^{13} \mathrm{C}$ NMR $\left(\mathrm{CDCl}_{3}\right): \delta=108.6(\mathrm{CH}-5), 124.0,126.0(\mathrm{Cl}-\mathrm{Ph} 2 \mathrm{CH}), 126.4(p-\mathrm{Ar} \mathrm{CH}), 127.0(\mathrm{Cl}-\mathrm{Ph} \mathrm{C}), 127.6$, 128.0 (Ar 2CH), 128.40, 128.60, 130.0, 130.4 (Ar CH), 131.4 (Ph C), 132.2 (C-6a), 134.2 (C10a), 135.2 (Cl-N-Ph C), 148.0 (C-4a), 154.0 (C-3), 156.0 (C-10b), 185.0 (C-6). - MS (EI, 70 $\mathrm{eV}): m / z(\%)=384[\mathrm{M}+1](32), 383\left[\mathrm{M}^{+}\right]$(100), 381 (28), 348 (20), 346 (30), 272 (34), 270 (38), 
196 (28), 194 (24), 118 (30), 116 (34), 77 (42). - $\mathrm{C}_{23} \mathrm{H}_{14} \mathrm{ClN}_{3} \mathrm{O}$ (383.84): Calcd. C, 71.97; H, 3.68; Cl, 9.24; N, 10.95. Found C, 71.82; H, 3.60; Cl, 9.16; N, 11.08.

\section{Reaction of amidrazones 1a-d with 10 and 15. General procedure}

A $250 \mathrm{ml}$ two necked bottom flask was flame-dried under $\mathrm{N}_{2}$ atmosphere and then cooled to room temperature Under $\mathrm{N}_{2}$ atmosphere, dry DMF $(30 \mathrm{~mL})$ containing a mixture of 1a-d (1 mmol) and 10 or 15 ( $1 \mathrm{mmol})$ is placed. The mixture was stirred with reflux (the reaction was followed by TLC analysis). The solvent was evaporated to its half volume. The obtained products 12 and 16 were filtered and recrystallized from the stated solvents.

5,6-Dichloro-3-phenyl-1H-indazole-4,7-dione (12). Red crystals $(0.22 \mathrm{~g}, 75 \%) ; R_{f}=0.3$ $\left(\mathrm{CH}_{2} \mathrm{Cl}_{2}\right)$, m.p. $280{ }^{\circ} \mathrm{C}$ (ethyl acetate). - IR (KBr): $v=3220(\mathrm{NH}), 3070-2996(\mathrm{Ar}-\mathrm{CH}), 1700$ $1690(\mathrm{C}=\mathrm{O}), 1610,1600(\mathrm{C}=\mathrm{N}), 1590(\mathrm{C}=\mathrm{C}) \mathrm{cm}^{-1}$. $-\mathrm{UV}\left(\mathrm{CH}_{3} \mathrm{CN}\right): \lambda_{\max }(\log \varepsilon)=410(4.1) .-{ }^{1} \mathrm{H}$ $\operatorname{NMR}\left(\mathrm{CDCl}_{3}\right): \delta=7.00-7.28(\mathrm{~m}, 5 \mathrm{H}, \mathrm{Ph}-\mathrm{H}) .12 .40(\mathrm{~s}, 1 \mathrm{H}, \mathrm{NH}) .-{ }^{13} \mathrm{C} \mathrm{NMR}\left(\mathrm{CDCl}_{3}\right): \delta=124.5$, (C-5), 126.8 (C-6), 127.2 (p-Ph CH), 127.6, 128.4 (Ar 2CH), 130.0 (Ph C), 132.0 (C-3a), 133.0 (C-7a), 155.8 (C-3), 175.8 (C-4), 176.4 (C-9). - MS (EI, $70 \mathrm{eV}): \mathrm{m} / \mathrm{z}(\%)=295$ [M+2] (12), 294 [M+1] (34), $293\left[\mathrm{M}^{+}\right]$(100), 258 (30), 256 (34), 224 (34), 222 (28), 218 (34), 120 (22), 118 (28), 80 (44), 77 (34). - $\mathrm{C}_{13} \mathrm{H}_{6} \mathrm{Cl}_{2} \mathrm{~N}_{2} \mathrm{O}_{2}$ (293.11): Calcd. C, 53.27; H, 2.06; Cl, 24.19; N, 9.56. Found: C, 53.16; H, 2.00; Cl, 24.10; N, 9.50.

3-Phenyl-1H-benzo[f]indazole-4,9-dione (16). Red crystals $(0.22 \mathrm{~g}, 80 \%) ; R_{f}=0.5\left(\mathrm{CH}_{2} \mathrm{Cl}_{2}\right)$, m.p. $250{ }^{\circ} \mathrm{C}$ (ethyl acetate). - IR (KBr): $v=3200(\mathrm{NH}), 3120-2990(\mathrm{Ar}-\mathrm{CH}), 1700-1685(\mathrm{C}=\mathrm{O})$, 1610, $1600(\mathrm{C}=\mathrm{N}), 1590(\mathrm{C}=\mathrm{C}) \mathrm{cm}^{-1}$. - UV $\left(\mathrm{CH}_{3} \mathrm{CN}\right): \lambda_{\max }(\log \varepsilon)=420(4.16) .-{ }^{1} \mathrm{H} \mathrm{NMR}$ $\left(\mathrm{CDCl}_{3}\right): \delta=7.10-7.36(\mathrm{~m}, 7 \mathrm{H}, \mathrm{Ph}-\mathrm{H}) .7 .74(\mathrm{dd}, 2 \mathrm{H}, J=8.0,1.2 \mathrm{~Hz}, \mathrm{Ar}-\mathrm{H}), 12.30(\mathrm{~s}, 1 \mathrm{H}, \mathrm{NH})$.${ }^{13} \mathrm{C} \mathrm{NMR}\left(\mathrm{CDCl}_{3}\right): \delta=126.8(p-\mathrm{Ph} \mathrm{CH}), 127.8,128.2(\mathrm{Ar} 2 \mathrm{CH}), 128.4,128.8,129.6,130.0(\mathrm{Ar}$ $\mathrm{CH}), 130.4$ (C-4a), 130.8 (Ph C), 132.4 (C-3a), 132.0 (C-8b), 134.2 (C-8a), 156.0 (C-3), 175.8 (C-4), 176.8 (C-9). - MS (EI, $70 \mathrm{eV}): m / z(\%)=274\left[\mathrm{M}^{+}\right](100), 196$ (30), 156 (60), 120 (22), 118 (34), 80 (40), 77 (36). - $\mathrm{C}_{17} \mathrm{H}_{10} \mathrm{~N}_{2} \mathrm{O}_{2}$ (274.28): Calcd. C, 74.45; H, 3.67; N, 10.21. Found: C, $74.36 ; \mathrm{H}, 3.60 ; \mathrm{N}, 10.10$.

\section{References and Notes}

1. (a) Zeman, E. M.; Brown, J. M.; Lemmon, M. J.; Hirst, V. K.; Lee, W. W. Int. J. Radiat. Onc. Biol. Phys. 1986, 12, 1239. (b) Zeman, E. M.; Baker, M. A.; Lemmon, M. J.; Pearson, C. I.; Adams, J. A.; Brown, J. M.; Lee, W. W.; Tracy, M. Int. J. Radiat. Onc. Biol. Phys. 1989, 16, 977. (c) Brown, J. M. Brit. J. Cancer 1993, 67, 1163.

2. Edwards, D. I.; Tocher, J. H.; Dale, L. D.; Widdick, D.; Virk, N. S. NATO ASI Ser., A 1990, 198, 275.

3. Daniels, J. S.; Gates, K. S. J. Am. Chem. Soc. 1996, 118, 3380.

4. Laderoute, K.; Wardman, P.; Rauth, A. M. Biochem. Pharmacol. 1988, 37, 1487.

5. Tocher, J. H.; Virk, N. S.; Edwards, D. I. Biochem. Pharmacol. 1990, 39, 781.

6. Mason, J. C.; Tennant, G. J. Chem. Soc. B 1970, 911. 
7. Arndt, F. Ber. 1913, 46, 3522.

8. Seng, F.; Ley, K. Angew. Chem. Int. Ed. 1972, 11, 1009.

9. Suzuki, H.; Kawakami, T. Synthesis 1997, 855.

10. Neilson, D. G.; Roger, R. M.; Heatle, J. W.; Newlands, L. R. Chem. Rev. 1970, 70, 151.

11. Modzelewska-Banachiewicz, B.; Matysiak, J.; Niewiadomy, A. Eur. J. Med. Chem. 2001, $36,75$.

12. Haldeman, R. G.; Morin, L. T.; Matsuda, K. (to American Cyanamid Co.) U. S. Patent 1963, 3,075,013; Chem. Abstr. 1963, 58, 11276 b.

13. Clemens, F.; Drutkowski, G.; Wiese, M.; Frohberg, P. Biochim. Biophys. Acta 2001, 1549, 88.

14. Schmidt, P.; Druey, J. Helv. Chim. Acta 1955, 38, 1560.

15. Katritzky, A. R.; Khashab, N. M.; Kirichenko, N.; Singh, A. J. Org. Chem. 2006, 71, 9051.

16. (a) Mamolo, M. G.; Falagiani, V.; Vio, L.; Banfi, E. Farmaco 1999, 54, 761. (b) Mamolo, M. G.; Vio, L.; Banfi, E.; Predominato, M.; Fabris, C.; Asaro, F. Farmaco 1992, 47, 1055.

(c) Banfi, E.; Mamolo, M. G.; Vio, L.; Predominato, M. J. Chemother. 1993, 5, 164. (d) Mamolo, M. G.; Vio, L.; Banfi, E.; Predominato, M.; Fabris, C.; Asaro, F. Farmaco 1993, 48, 529. (e) Mamolo, M. G.; Vio, L.; Banfi, E. Farmaco 1996, 51, 65.

17. (a) Gokhale, N.; Padhye, S.; Rathbone, D.; Billington, D.; Lowe, P.; Schwalbe, C.; Newton, C. Inorg. Chem. Commun. 2001, 4, 26. (b) Gokhale, N. H.; Padhye, S. S.; Padhye, S. B.; Anson, C. E.; Powell, A. K. Inorg. Chim. Acta 2001, 319, 90.

18. Echavarren, A. M. J. Org. Chem. 1990, 55, 4255.

19. Villacampa, M.; Perez, J. M.; Avendano, C.; Menendez, J. C. Tetrahedron 1994, 50, 10047.

20. Altuna-Urquijo, M.; Stanforth, S. P.; Tarbit, B. Tetrahedron Lett. 2005, 46, 6111.

21. Drutkowski, G.; Donner, C.; Schulze I.; Frohberg, I. Tetrahedron 2002, 58, 5317.

22. Boivin, J.; Husinec, S.; Zard, S. Z. Tetrahedron 1995, 51, 11737.

23. Aly, A. A.: NourEl-Din, A. M.; Gomaa, M.-A.; Brown, A. B.; Fahmi, M. S. J. Chem. Res. 2007, 439.

24. Aly, A. A.; NourEl-Din, A. M.; Gomaa, M.-A.; Fahmi, M. S. Z. Naturforsch. 2008, 63B, in press.

25. Aly, A. A.; Gomaa, M.-A.; NourEl-Din, A. M.; Fahmi, M. S. Z. Naturforsch. 2006, 61B, 1239.

26. (a) Deng, X.; Mani, N. S. Org. Lett. 2006, 8, 3505. (b) Huisgen, R. Angew. Chem. Int. Ed. 1963, 2, 256. (c) Huisgen, R. In 1,3-Dipolar Cycloaddition; Padwa, A., Ed.; WileyInterscience: New York, 1984; p 1. (d) Gribble, G. W. J. Chem. Soc., Perkin Trans. 1 2000, 1045.

27. Singh, M. W.; Karmakar, A.; Barooah, N.; Baruah, J. B. Beilstein J. Org. Chem. 2007, 3, 10.

28. Hassan, A. A.; Aly, A. A.; El-Sheref, E. M. Arkivoc 2007, xiv, 229. 